\title{
Modification of the local atomic structure around Mn atoms in (Ga, Mn)As layers by high temperature annealing
}

\author{
I N Demchenko ${ }^{1}, \mathrm{~K}_{\text {Lawniczak-Jablonska }}{ }^{1}, \mathrm{~T} \mathrm{Story}^{1}, \mathrm{~V}_{\text {Osinniy }}{ }^{1}$, \\ R Jakiela ${ }^{1}$, J Z Domagala ${ }^{1}$, J Sadowski ${ }^{1}$, M Klepka ${ }^{1}$, A Wolska ${ }^{1}$ and \\ M Chernyshova ${ }^{2}$ \\ ${ }^{1}$ Institute of Physics PAS, Aleja Lotników 32/46, 02-668, Warsaw, Poland \\ ${ }^{2}$ Institute of Plasma Physics and Laser Microfusion, 23 Hery Street, 01-497 Warsaw, Poland \\ E-mail: demch@ifpan.edu.pl
}

Received 5 September 2007, in final form 12 October 2007

Published 12 November 2007

Online at stacks.iop.org/JPhysCM/19/496205

\begin{abstract}
Changes of the local structure around Mn atoms in ( $\mathrm{Ga}, \mathrm{Mn})$ As layers after high temperature annealing were determined by x-ray absorption spectroscopy (EXAFS) and high-resolution x-ray diffraction (HRXRD) measurements, and related to their magnetic properties. X-ray absorption is known to be able to detect crystalline structure changes occurring around investigated atoms. The qualitative and quantitative analysis of EXAFS spectra gives unambiguous evidence for the transition from a cubic to a hexagonal phase around $\mathrm{Mn}$ atoms as a result of annealing at temperatures of 500 and $600{ }^{\circ} \mathrm{C}$. The performed HRXRD investigation indicated a relaxation of the GaAs matrix during annealing, from highly to slightly compressively strained, and finally, after the formation of inclusions of hexagonal MnAs, to slightly tensile strained. The sample with hexagonal MnAs inclusions exhibits ferromagnetic properties up to room temperature with almost all $\mathrm{Mn}$ atoms being in a ferromagnetic phase.
\end{abstract}

\section{Introduction}

Recently there has been rapid development in the field of research called spintronics. The goal of these studies is to create conceptually new devices which will utilize the spin of an electron. To achieve this goal it is necessary to fabricate effective injectors of polarized spins, learn how to manipulate and detect spin polarization, and we must integrate magnetic materials with conventional semiconductor technology. All of this requires the fabrication of suitable materials with appropriate properties at room temperature and the manufacture of a reliable spin based device.

Mn doped GaAs is the most comprehensively studied material for semiconductor spintronics. $\mathrm{Ga}_{1-x} \mathrm{Mn}_{x}$ As exhibits ferromagnetic properties for Mn content exceeding $x=$ 
0.005 [1-3]. (Ga, Mn)As with a Mn content of several atomic per cent can be grown by molecular beam epitaxy (MBE). To avoid MnAs segregation, the MBE growth of (Ga, Mn)As must be performed at temperatures which are much lower than those normally applied (550$650{ }^{\circ} \mathrm{C}$ ) for $\mathrm{GaAs}$, i.e. at $180-300^{\circ} \mathrm{C}$, depending on $\mathrm{Mn}$ content [2]. If we substitute $\mathrm{Mn}^{2+}$ ions with $5 / 2$ spin for $\mathrm{Ga}$ in GaAs, the substituted $\mathrm{Mn}^{2+}$ are ferromagnetically coupled due to exchange interactions with valence band holes, which gives rise to the ferromagnetic behavior of $\mathrm{Ga}_{1-x} \mathrm{Mn}_{x}$ As. The substitutional $\mathrm{Mn}$ ions $\left(\mathrm{Mn}_{\mathrm{Ga}}\right)$ act as acceptors, generating holes that mediate the ferromagnetic exchange [4]. However, one must be careful, low (MBE) growth temperature can lead to high density of point defects [5, 6], the most important of these defects are now known as As-anti-sites $\left(\mathrm{As}_{\mathrm{Ga}}\right)$ [7], and $\mathrm{Mn}$ interstitials $\left(\mathrm{Mn}_{\mathrm{i}}\right)$ [8] which are double donors significantly compensating a fraction of free holes.

Solid solutions of $\mathrm{Ga}_{1-x} \mathrm{Mn}_{x}$ As with $x \approx 0.05$ reveal ferromagnetic properties having Curie temperatures of about $T_{\mathrm{C}} \approx 110 \mathrm{~K}$ [9]. Up to now, the highest ferromagnetic phase transition temperature reported for $\mathrm{Ga}_{1-x} \mathrm{Mn}_{x} \mathrm{As}$ with $x \sim 0.08$ is close to $170 \mathrm{~K}$ [10]. This material was grown as thin films, with a thickness below $0.1 \mu \mathrm{m}$, and annealed at low temperatures after the growth. Moreover, it can be noticed that $T_{\mathrm{C}}$ can reach up to $250 \mathrm{~K}$ in GaAs based structures with a $\delta\langle\mathrm{Mn}\rangle$ doped layer [11]. It is known that the Curie temperature increases with both Mn content and hole concentration. The maximum Mn content reported for uniform $\mathrm{Ga}_{1-x} \mathrm{Mn}_{x}$ As alloy is close to $x=0.1$, but very recently for the case of extremely thin layers (thickness below $10 \mathrm{~nm}$ ) a content was reported of $x=0.2$ [12]. Increasing the Mn content in $\mathrm{Ga}_{1-x} \mathrm{Mn}_{x}$ As requires a reduction of the growth temperature. For $\mathrm{Ga}_{1-x} \mathrm{Mn}_{x}$ As with $x=0.01$ of $\mathrm{Mn}$ the maximum growth temperature is close to $300^{\circ} \mathrm{C}$, but to avoid separation of MnAs clusters it must be reduced below $200^{\circ} \mathrm{C}$ for Mn content $x \geqslant 0.08$ [12-14].

Higher $T_{\mathrm{C}}$ can be achieved for materials where the compensating defects have been removed by using low temperature post-growth annealing procedures. In [15], it was shown that a second MnAs phase, with NiAs structure, appears with the increase of either the Mn flux or the substrate temperature or both of these parameters above the segregation threshold.

On the other hand, MnAs clusters with considerable dimensions can be produced from single-phase GaMnAs material by high-temperature post-growth annealing at temperatures higher than $500^{\circ} \mathrm{C}$, which results in multiphase materials [16].

Up to now these precipitations were considered as a drawback, however, since MnAs is a metallic ferromagnet, with a $T_{\mathrm{C}}$ of about $318 \mathrm{~K}$, the GaAs:MnAs granular system constitutes an interesting material with small ferromagnetic nanoparticles immersed in the semiconductor host lattice. Such a composite material could be considered as a good semiconductor (applicable for integrated circuits) filled with small magnets, thus providing a built-in magnetic field at room temperature. This field would affect the semiconductor band structure, in particular, leading to differentiation of semiconductor spin states, which is essential for a spintronic material. Therefore, an attractive method to control the spin polarization may be achieved for two phases of a material homogeneously distributed, magnetic and semiconducting at the same time.

In the present paper we have studied the $\mathrm{Ga}_{0.95} \mathrm{Mn}_{0.05} \mathrm{As}$ film before and after annealing at a temperatures of 500 and $600{ }^{\circ} \mathrm{C}$. EXAFS spectra were examined to determine the average local atomic structure around $\mathrm{Mn}$ atoms. The EXAFS technique is sensitive to the local structure around a selected element, which makes it an effective tool for studying multicomponent materials. In the literature, several EXAFS studies for $\mathrm{Mn} / \mathrm{GaAs}$ digital alloys [17-19], Mn $\delta$-doped GaAs [20], and $\mathrm{Ga}_{1-x} \mathrm{Mn}_{x}$ As films ( $x=0.005$ and 0.074) [21] have been reported. Out of all of the reported structures for as-grown films, EXAFS data indicate that Mn atoms are the most likely substitute for $\mathrm{Ga}$ in a GaAs matrix. Dramatic structural changes were reported at a high annealing temperature (around $450^{\circ} \mathrm{C}$ ), e.g. [19]. The authors suggested that this was 
probably due to initial stages of precipitation of an MnAs phase. To verify this hypothesis we have performed detailed studies of $\mathrm{Ga}_{0.95} \mathrm{Mn}_{0.05} \mathrm{As}$ single layers annealed at 500 and $600{ }^{\circ} \mathrm{C}$. To characterize the layers, SIMS and XRD methods were used to evaluate the content of Mn and quality of the grown layer. Measurements of the magnetic moment for the same samples were also performed to relate the structural properties with the magnetic ones.

\section{Experimental details}

The $\mathrm{Ga}_{0.95} \mathrm{Mn}_{0.05}$ As layers ( $0.91 \mu \mathrm{m}$ thick) were grown by using the MBE technique on semiinsulating $\mathrm{GaAs}(100)$ substrates at $230{ }^{\circ} \mathrm{C}$ and then were covered with a $0.2 \mu \mathrm{m}$ thick layer of amorphous As. The GaMnAs samples were then removed from the vacuum system, cleaved into several pieces, and reintroduced into the MBE system for high temperature annealing procedures. The amorphous As layer was deposited to prevent the GaMnAs surface from being exposed to air, thus avoiding possible surface effects associated with the presence of an oxidized GaMnAs surface. The protecting As layer was desorbed at the early stage of the high temperature annealing, revealing the smooth two-dimensional surface of initial GaMnAs. To avoid surface roughening which occurs at high annealing temperatures, the annealing was performed in an As flux. The surfaces of the annealed samples were controlled by reflection high energy electron diffraction (RHEED) and shown to be smooth, with two-dimensional streaky diffraction patterns throughout the whole high temperature annealing procedure.

Two pieces of the investigated sample were annealed at temperature $500^{\circ} \mathrm{C}$ ' $a$ ' and $600{ }^{\circ} \mathrm{C}$ ' $c$ ', respectively, for 30 min to investigate the thermally induced changes in the local structure around the Mn atoms. No heat treated ('as-grown') sample was used as a reference. Further details of the growth procedure are given in [22].

The content of $\mathrm{Mn}, x$, in the initial $\mathrm{Ga}_{1-x} \mathrm{Mn}_{x}$ As layers was investigated by comparing the growth rates for low temperature GaAs and GaMnAs through RHEED intensity oscillations during the GaMnAs MBE growth process, and further verified by secondary ion mass spectrometry (SIMS) using a CAMECA IMS6F microanalyzer. SIMS measurements were performed with an oxygen $\left(\mathrm{O}_{2}^{+}\right)$primary beam, with the beam current kept at $700 \mathrm{nA}$. The size of the eroded crater was about $150 \mu \mathrm{m} \times 150 \mu \mathrm{m}$ and the secondary ions were collected from a central region of $30 \mu \mathrm{m}$ in diameter. Mn concentrations were derived from the intensity of $\mathrm{Mn}^{+}$species, with the $\mathrm{As}^{+}$matrix signal being used as a reference, and a $1 \times 10^{16} \mathrm{~cm}^{-2}$ dose of Mn implanted on GaAs was used as a calibration standard.

To examine the possibility of phase separation after annealing the HRXRD measurements were carried out using a high-resolution diffractometer (Philips X'Pert-MRD) equipped with a parabolic X-ray mirror, a four-bounce Ge 220 monochromator at the incident beam, and a three-bounce $\mathrm{Ge}$ analyzer at the diffracted beam.

EXAFS measurements were performed at liquid nitrogen temperature, at the A1 experimental station in HASYLAB (Hamburg, Germany) using the double crystal Si(111) monochromator. The K-edge spectra of Mn $(6539 \mathrm{eV})$ were registered using a seven-element fluorescence Si detector.

The magnetic properties were studied in the temperature range from 5 to $280 \mathrm{~K}$ using a superconducting quantum interference device (SQUID) magnetometer, where an external magnetic field of up to 2000 Oe was applied in the plane of the layer.

\section{Results and discussion}

The concentration of $\mathrm{Mn}$ in all of the examined samples was at $x \approx 0.05$ and was not significantly changed during the annealing process. Figure 1 shows the SIMS profiles for the 


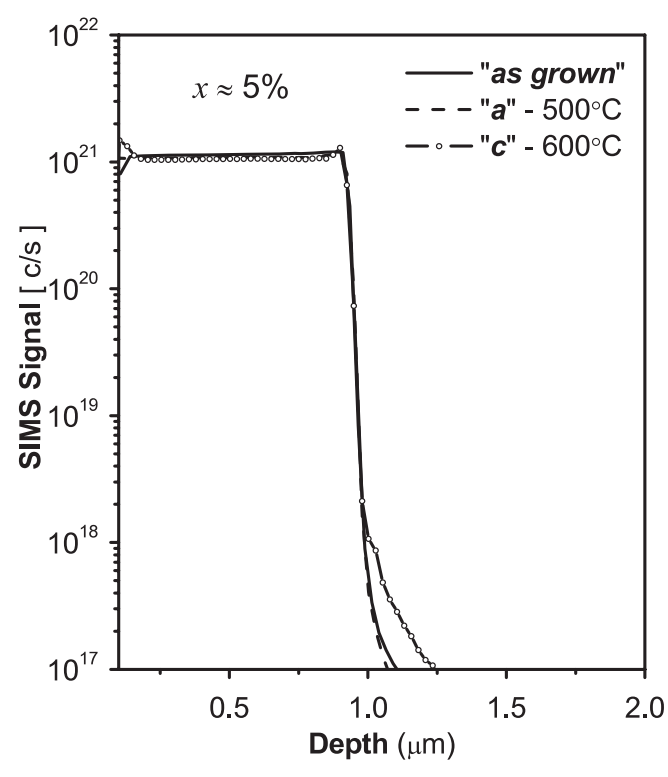

Figure 1. SIMS profiles of the Mn distribution along the growth direction for 'as-grown' and annealed GaMnAs layers.

Mn distribution over the thickness of $\mathrm{Ga}_{0.95} \mathrm{Mn}_{0.05} \mathrm{As}$ layers; the thickness of the examined layer was measured by an Alpha-Step Profiler.

HRXRD measurements indicated changes in the lattice constant of the examined GaMnAs samples upon annealing. The $2 \Theta / \omega$ scans across the 004 symmetric reflection are shown in figure 2. The thin line corresponds to the 'as-grown' sample, the thick line to the sample annealed at $500^{\circ} \mathrm{C}\left({ }^{\prime} a\right.$ '), and the dashed line to the sample annealed at $600^{\circ} \mathrm{C}\left({ }^{\circ} c\right.$ '). In addition to the GaAs substrate peak (at $2 \theta \approx 66.05^{\circ}$ ), an intense peak corresponding to the $\mathrm{Ga}_{0.95} \mathrm{Mn}_{0.05} \mathrm{As}$ layer is observed at $2 \theta \approx 65.80^{\circ}$ for the 'as-grown' sample. Upon annealing, the diffraction peak at $\approx 65.80^{\circ}$ disappears and new peaks appear at the lower $\left(2 \theta \approx 66.03^{\circ}\right.$ for sample ' $a$ ') and higher $\left(2 \theta \approx 66.07^{\circ}\right.$ for sample ' $c$ ') angles of diffraction around the GaAs substrate peak. Similar results were presented in [23]. The presence of the separated peak for the layer in the 'as-grown' sample indicates the existence of highly compressive strain in the layer $(\Delta a / a)=0.65 \%$. During annealing, the matrix relaxes and only slight compressive strain remains in the layer for sample ' $a$ ' $(\Delta a / a=0.056 \%)$. For sample ' $c$ ' $(\Delta a / a=-0.067 \%)$ it is changed to a slight tensile strain. New peaks corresponding to a new phase formation (i.e. Mn-related clusters/inclusions) were not observed around 004 reflection.

Upon annealing, the Mn atoms leave the zinc-blende lattice to form clusters embedded in the GaAs matrix. To check the local structure around Mn atoms, quantitative analysis of the EXAFS spectra for investigated samples was performed. The Athena and Artemis programs (based on the IFEFFIT library of numerical and XAS algorithms) were applied [24]. The theoretical amplitude and phases were calculated using the FEFF8 program [25, 26].

To keep the systematic errors in the data analyzing procedure at the same level, all the analyzed data were worked out in the same way. The EXAFS oscillations $\chi(k)$ shown in figure 3 were extracted from the experimental data using standard procedures [27]. The preedge background was subtracted with a linear function whereas an $R_{\mathrm{bkg}}$ parameter equal to $1.1 \AA$ was used for the atomic background removal. The Fourier transform was computed using the Kaiser-Bessel filtering function. The $k^{1}$ weighted $\chi(k)$ data were Fourier transformed (FFT) in the $k$ range between 3.28 and $12.48 \AA^{-1}$ for all the examined samples. The modulus of the Fourier-transformed $k \chi(k)$ functions $(|\chi(R)|)$ is presented in figure 4 . The changes of the 


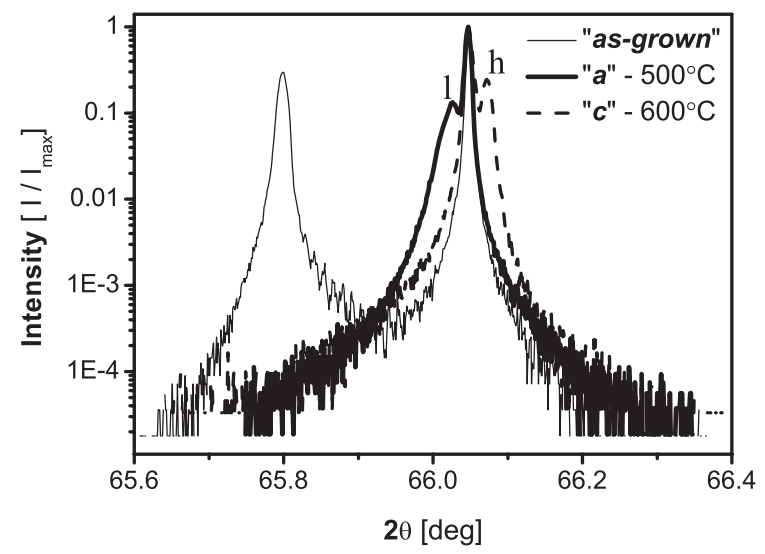

Figure 2. High-resolution $2 \Theta / \omega$ XRD scans across 004 symmetric Bragg reflection in $\mathrm{Ga}_{0.95} \mathrm{Mn}_{0.05} \mathrm{As} / \mathrm{GaAs}(100)$ samples, recorded before (thin line) and after annealing at the temperatures $500^{\circ} \mathrm{C}$ (' $a$ '-thick line) and $600{ }^{\circ} \mathrm{C}$ (' $c$ ' - dashed line). 1 and $\mathrm{h}$ mark the peaks at low and high angles of diffraction.

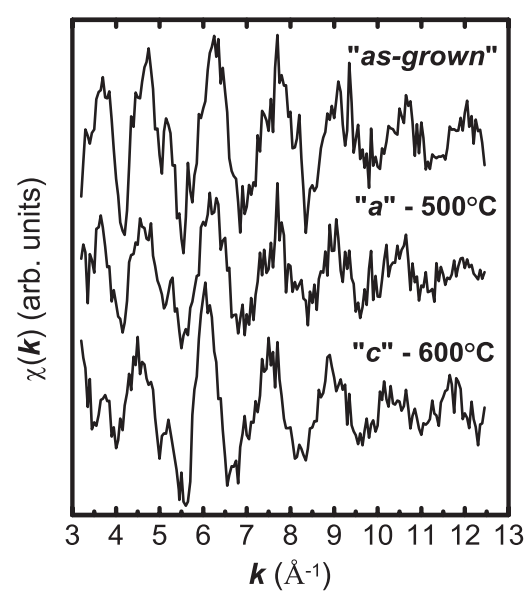

Figure 3. EXAFS oscillations $\chi(k)$ for the $\mathrm{Ga}_{0.95} \mathrm{Mn}_{0.05} \mathrm{As} / \mathrm{GaAs}(100)$ samples (from top to bottom): 'as-grown', annealed at temperature $500^{\circ} \mathrm{C}-$ ' $a$ ' and $600^{\circ} \mathrm{C}-$ ' $c$ '.

local structure around $\mathrm{Mn}$ atoms due to the annealing are clearly visible. The radial distribution of atoms in two of the nearest coordination shells around Mn for the 'as-grown' sample (thick line) is similar to that for sample ' $a$ ' (dotted line). Additionally, some amplitude reduction of the peak corresponding to the first (about 26\%) and the second (about 29\%) coordination shells can be noticed for the sample ' $a$ ' in comparison with the 'as-grown' one. A similar effect was observed in [19] and was related to the reduction of the coordination number of As atoms due to the initial stages of the MnAs phase precipitation. Moreover, it is evident that the $|\chi(R)|$ function corresponding to the sample ' $c$ ' annealed at $600^{\circ} \mathrm{C}$ is very different from those for the 'as-grown' and for ' $a$ ' samples for higher $R$. It may indicate that some structural changes around $\mathrm{Mn}$ atoms occurred after annealing at $600{ }^{\circ} \mathrm{C}$.

As is well known, the position of $\mathrm{Mn}$ atoms in the zinc-blende lattice of $\mathrm{Ga}_{1-x} \mathrm{Mn}_{x} \mathrm{As}$ has a crucial role in determining the magnetic properties of the material. Substitutional Mn atoms occupying the gallium sites $\left(\mathrm{Mn}_{\mathrm{Ga}}\right)$ act as acceptors and contribute to the ferromagnetic ordering. On the other hand, manganese atoms in the interstitial positions $\left(\mathrm{Mn}_{\mathrm{i}}\right)$ are double 


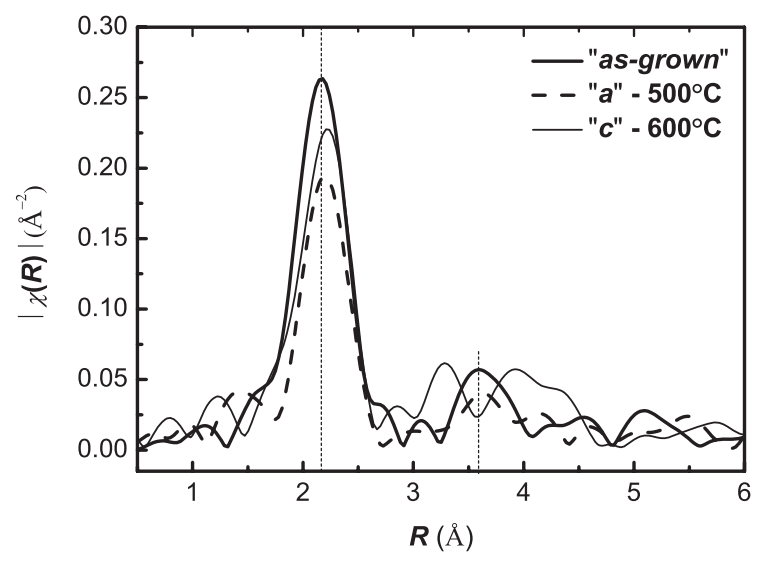

Figure 4. Modulus of the Fourier-transformed $k \chi(k)$ functions corresponding to investigated samples: 'as-grown' (thick line), ' $a$ ' $-500^{\circ} \mathrm{C}$ (dotted line), and ' $c$ ' $-600^{\circ} \mathrm{C}$ (thin line).

Table 1. The local structural parameters for five considered models of Mn coordination ( $N$ is coordination number, $R$-interatomic distance).

\begin{tabular}{|c|c|c|c|}
\hline Model structure & Neighboring atom & $N$ & $R(\AA)$ \\
\hline \multirow{7}{*}{$\begin{array}{l}\text { Mn in GaAs substitutional model, 'Mn } \mathrm{Ma}_{\mathrm{Ga}} \text { ' and * for } \\
\text { 'Mn } \mathrm{Ga}_{\mathrm{G}-\text { shift' model (see the text) }}\end{array}$} & As & 4 & 2.448 \\
\hline & & & $2.550^{*}$ \\
\hline & $\mathrm{Ga}$ & 12 & 3.998 \\
\hline & As & 12 & 4.688 \\
\hline & $\mathrm{Ga}$ & 6 & 5.653 \\
\hline & As & 12 & 6.161 \\
\hline & $\mathrm{Ga}$ & 24 & 6.924 \\
\hline \multirow{6}{*}{$\begin{array}{l}\mathrm{Mn} \text { in GaAs interstitial model where there are four As } \\
\text { atoms around } \mathrm{Mn} \text { absorbing atom in the first } \\
\text { coordination shell, ' } \mathrm{Mn}_{\mathrm{i}(\mathrm{As})} \text { ' }\end{array}$} & As & 4 & 2.448 \\
\hline & $\mathrm{Ga}$ & 6 & 2.827 \\
\hline & As & 12 & 4.688 \\
\hline & $\mathrm{Ga}$ & 8 & 4.896 \\
\hline & As & 12 & 6.161 \\
\hline & $\mathrm{Ga}$ & 24 & 6.321 \\
\hline \multirow{6}{*}{$\begin{array}{l}\mathrm{Mn} \text { in GaAs interstitial model where there are four } \mathrm{Ga} \\
\text { atoms around } \mathrm{Mn} \text { absorbing atom in the first } \\
\text { coordination shell, ' } \mathrm{Mn}_{\mathrm{i}(\mathrm{Ga})} \text {, }\end{array}$} & $\mathrm{Ga}$ & 4 & 2.448 \\
\hline & As & 6 & 2.827 \\
\hline & $\mathrm{Ga}$ & 12 & 4.688 \\
\hline & As & 8 & 4.896 \\
\hline & $\mathrm{Ga}$ & 12 & 6.161 \\
\hline & As & 24 & 6.321 \\
\hline \multirow[t]{6}{*}{ MnAs hexagonal,'MnAs’ } & As & 6 & 2.577 \\
\hline & $\mathrm{Mn}$ & 2 & 2.851 \\
\hline & $\mathrm{Mn}$ & 6 & 3.718 \\
\hline & As & 6 & 4.524 \\
\hline & $\mathrm{Mn}$ & 12 & 4.685 \\
\hline & As & 6 & 4.785 \\
\hline
\end{tabular}

donors and decrease the hole concentration, thus destroying ferromagnetism. To obtain more quantitative information for the investigated samples, the fitting procedures were performed in the $R$ ranges $1.3-4.80 \AA, 1.7-4.41 \AA$, and 1.4-4.80 $\AA$ for 'as-grown', ' $a$ ' and ' $c$ ' samples respectively. Five geometrical models were considered for the construction of the theoretical EXAFS signal (the nearest six coordination shells around Mn atoms for the models are presented in table 1). The following models were considered: ' $\mathrm{Mn}_{\mathrm{Ga}}$ ', ' $\mathrm{Mn}_{\mathrm{Ga}}$ shift', ' $\mathrm{Mn}_{\mathrm{i}(\mathrm{As})}$, ' $\mathrm{Mn}_{\mathrm{i}(\mathrm{Ga})}$ ' and 'MnAs' (hexagonal). 

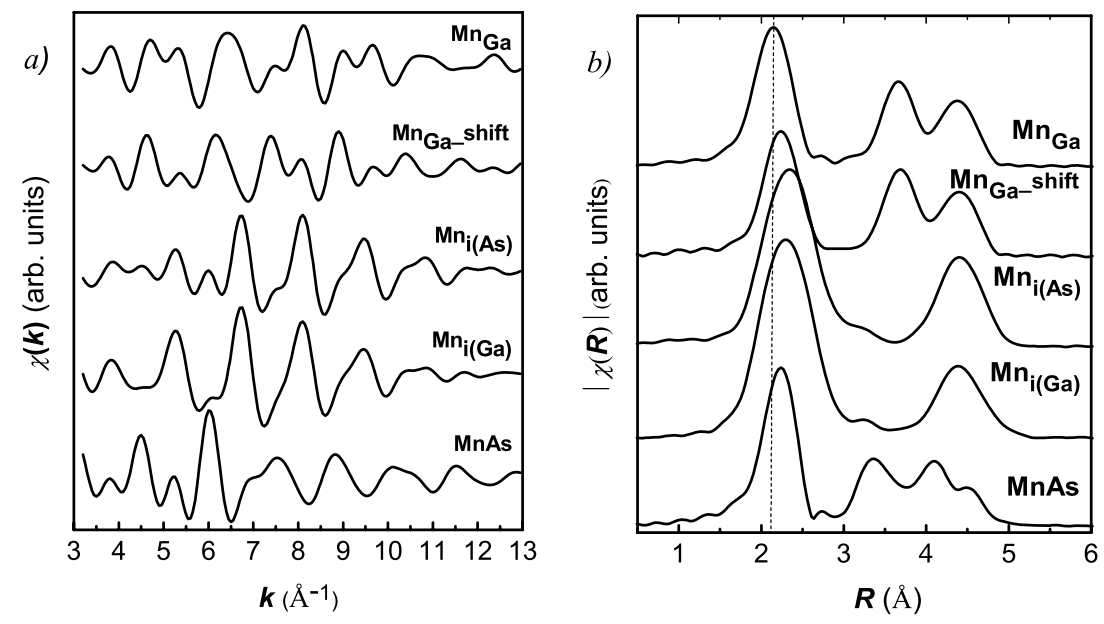

Figure 5. The results of the theoretical calculations using the parameters of the model structures listed in table 1: (a) EXAFS oscillations $\chi(k)$; (b) modulus of the Fourier-transform of $\chi(k)$.

In the substitution model ' $\mathrm{Mn}_{\mathrm{Ga}}$ ' the $\mathrm{Mn}$ atom is substituted into the Ga site in the GaAs host matrix, and a lattice distance of $a_{\mathrm{GaAs}}=5.6533 \AA$ and the $F \overline{4} 3 M$ space group were used. In the model marked as ' $\mathrm{Mn}_{\mathrm{Ga}-\mathrm{shift}}$ ', the $\mathrm{Mn}$ atoms in the first coordination shell were shifted at about $+0.1 \AA$ according to the ' $\mathrm{Mn}_{\mathrm{Ga}}$ ' model whereas all the other atoms (in the higher coordination shells) were fixed in the previous positions. Two interstitial models ' $\mathrm{Mn}_{\mathrm{i}(\mathrm{As})}$ ' and ' $\mathrm{Mn}_{\mathrm{i}(\mathrm{Ga})}$ ' were considered, the ' $\mathrm{Mn}_{\mathrm{i}(\mathrm{As})}$ ' model consists of an $\mathrm{Mn}$ atom entering the tetragonal interstitial site (at the $(1 / 2,1 / 2,1 / 2)$ position) in the GaAs lattice coordinated by four nearest neighbor As atoms. In the ' $\mathrm{Mn}_{\mathrm{i}(\mathrm{Ga})}$ ' model the $\mathrm{Mn}$ ion (at $(-1 / 4,-1 / 4,-1 / 4)$ position in the unit cell) is again at the interstitial site in the GaAs lattice, but it is coordinated by four Ga atoms, whereas, the 'MnAs' model corresponds to the hexagonal NiAs-type structure $\left(P 6_{3} / m m c\right.$ space group) with the lattice constants $a=3.718 \AA$ and $c=5.702 \AA$.

The normalized EXAFS oscillations $\chi(k)$ and modulus of their Fourier-transforms $(|\chi(R)|)$ for these five structural models are shown in figures 5(a) and (b). For each model a cluster with a radius of about $7 \AA$ was generated and theoretical calculations were performed, keeping scattering paths up to about $5 \AA$.

Comparing the various $|\chi(R)|$ curves (figure $5(\mathrm{~b})$ ) for different models one notices the similarity between 'interstitial' models, where the crystal structures are identical due to the similarity of the As and Ga backscattering parameters because they only differ by 2 in $\mathrm{Z}$. Therefore, using EXAFS, one cannot distinguish between these two models. The first peak for the ' $\mathrm{Mn}_{\mathrm{i}(\mathrm{As})}$ ' and ' $\mathrm{Mn}_{\mathrm{i}(\mathrm{Ga})}$ ' models is broader in comparison with the ' $\mathrm{Mn}_{\mathrm{Ga}}$ ' and ' $\mathrm{Mn}_{\mathrm{Ga}} \mathrm{shift}_{\text {' }}$ models and is shifted towards larger $R$. This is due to the existence of two scattering paths (see table 1) in the 'interstitial' models with $R$ values 2.448 and $2.827 \AA$, which are not resolved in the radial distribution function, and in the 3-4 $\AA$ region, a gap is observed. While the ' $\mathrm{Mn}_{\mathrm{Ga}}$ ', ' $\mathrm{Mn}_{\mathrm{Ga}-\mathrm{s}}$ hift' models have second coordination shells close to $3.998 \AA$. The 'MnAs' model has the contribution of the third coordination shell around $3.718 \AA$. Comparing the ' $\mathrm{Mn}_{\mathrm{Ga}}$ ' and ' $\mathrm{Mn}_{\mathrm{Ga}}$ _shift' models, one observes in the ' $\mathrm{Mn}_{\mathrm{Ga} \_}$shift' model a decrease of about $10 \%$ for the first peak amplitude as well a shift of $0.1 \AA$ towards higher $R$ values. Obviously, the local structure around Mn atoms for the 'MnAs' model differs from the other models at higher $R$.

During the last few years, GaMnAs structures with different compositions of Mn were thoroughly examined by EXAFS [17-21], therefore we do not concentrate on the details of 


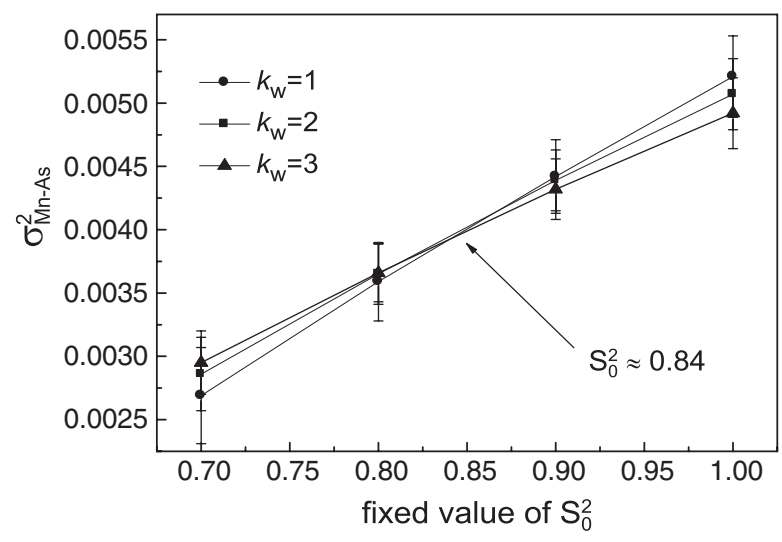

Figure 6. Determination of $S_{0}^{2}$ for the $\mathrm{K}$ edge of $\mathrm{Mn}$ in the $\mathrm{Ga}_{0.95} \mathrm{Mn}_{0.05} \mathrm{As}$ ' as grown' layer. Three lines form a small triangle around $S_{0}^{2} \approx 0.84$.

analysis for the 'as-grown' sample. Analysis for this sample was done as in [20], taking into account the multiple scattering but, additionally, the amplitude-damping factor $\left(S_{0}^{2}\right)$ was estimated. The $S_{0}^{2}$ factor was determined by data fitting the 'as-grown' sample using a well-known approach, which can be applied only when the first peak in the data is single (i.e. contains one kind of atom) [28]. The $S_{0}^{2}$ and $\sigma^{2}$ are highly correlated parameters, but $\sigma^{2}$ has a $k$ dependence in the EXAFS equation whereas $S_{0}^{2}$ does not. Therefore, these parameters affect the data in different ways when the $\chi(k)$ data is weighted differently. This difference in $k$ dependence can be exploited to break the correlation between these two parameters when the first peak is truly isolated from the other coordination shells. A series of fits were performed for the first shell for the data with different $k$-weights, assuming different values of $S_{0}^{2}$. The limit of the window used for spectral isolation of the first shell was 1.3-2.95 $\AA$. In each case a very simple fitting model was used, yielding the $\sigma_{\mathrm{Mn}-\mathrm{As}}^{2}$ versus $S_{0}^{2}$ dependence plotted in figure 6 . The value of $S_{0}^{2}=0.84$ common to all the fits was taken as an amplitude-damping factor and was kept constant in further analysis, thus limiting the number of fitting parameters.

A comparison between models (figure 5(a)) and measured functions $\chi(k)$ was performed (figures 7(a) and (b)) to find the best model for structures annealed at 500 and $600{ }^{\circ} \mathrm{C}$. The function $\chi(k)$ calculated for the ' $\mathrm{Mn}_{\mathrm{Ga}}$ shift' model appears to be closer to the experimental data for layer ' $a$ ' than $\chi(k)$ calculated for the ' $\mathrm{Mn}_{\mathrm{Ga}}$ ' model, which indicates that this model is closer to the atomic arrangement formed in this structure. Moreover, as was noticed above, the amplitude of the peak corresponding to the first coordination shell decreases by $10 \%$ (see figure 5(b)). This is due to the increase of the Debye-Waller (DW) factor from $0.0033\left({ }^{\circ} \mathrm{Mn}_{\mathrm{Ga}}\right.$ ' model) to $0.0035 \AA^{2}$ ('Mn ${ }_{\mathrm{Ga}}$ shift' model) as calculated by the FEFF program for both models. Finally, one might notice the high similarity between $\chi(k)$ oscillations observed in sample ' $c$ ' and the predications of the 'MnAs' model (figure 7(b)). Therefore, these two models were used as a starting point for EXAFS analysis for samples ' $a$ ' and ' $c$ '. The lack of similarity between the experimental spectra and the interstitial models allows us to conclude that there is not a significant number of $\mathrm{Mn}$ atoms occupying the interstitial positions in any of the considered samples. The results of the quantitative data analysis for all samples are reported in table 2 and figure 8.

Let us first consider the results of an EXAFS analysis for the 'as-grown' sample. The distances $R$ for three coordination shells are in good agreement with the results presented in [29] for $\mathrm{Ga}_{0.95} \mathrm{Mn}_{0.05} \mathrm{As}$, which are given for comparison in table 2. The Mn-As bond length $\left({ }^{1} R_{\mathrm{Mn}-\mathrm{As}}^{\mathrm{as}-\text { grown }}=2.496 \pm 0.003 \AA\right.$ ) for the first shell is longer (about $2 \%$ ) than the model value $2.448 \AA$ (see table 1 ). The distance to the second coordination shell ${ }^{2} R_{\mathrm{Mn}-\mathrm{Ga}}^{\mathrm{as}-\text { grown }}=$ 

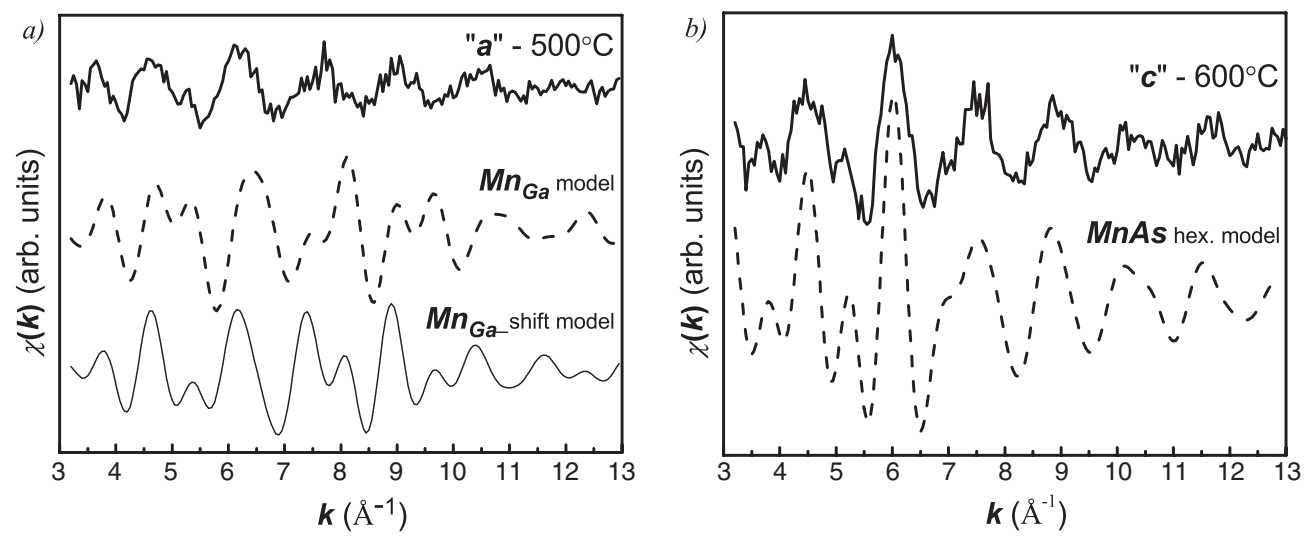

Figure 7. (a) Normalized EXAFS oscillations $\chi(k)$ observed experimentally in sample ' $a$ ' annealed at $500{ }^{\circ} \mathrm{C}$ and theoretically calculated for ' $\mathrm{Mn}_{\mathrm{Ga}}$ ' and ' $\mathrm{Mn}_{\mathrm{Ga}}$ shift' models; (b) the same curves corresponding to sample ' $c$ ' and the 'MnAs' model.

$4.03 \pm 0.01 \AA$ is close (within $0.7 \%$ ) to the model value of $3.998 \AA$ (table 1 ) while the distance to the third shell is within $1.2 \%\left({ }^{3} R_{\mathrm{Mn}-\mathrm{As}}^{\mathrm{as}-\text { grown }}=4.74 \pm 0.01 \AA\right.$ and the model value is $\left.4.688 \AA\right)$. This means that for the 'as-grown' sample, the local distortion is mainly affected by the first and third coordination shells (with As atoms only). Some differences in DW factors for the first and the second shells observed between our data and the results reported in [29] are related to thermal disorder, which was reduced in our case by measurements at liquid nitrogen temperature. The increase of the DW factor for the third shell of the examined sample can be associated with the increase of the static disorder in the investigated sample. In summary, the Mn local structure for the 'as-grown' sample was found to be similar to that of an Mn atom substituted into a Ga site within a GaAs lattice. The relatively good fit for this model indicates that only an insignificant fraction of $\mathrm{Mn}$ atoms can be located at the interstitial positions.

The results of EXAFS analysis for the 'as-grown' sample point at the homogeneity of this sample, and are in agreement with independent results obtained by transmission electron microscopy (TEM) for the same sample [30].

Let us discuss now sample ' $a$ '. The analysis of TEM images for this sample revealed Mn-rich zinc-blende $\mathrm{Mn}(\mathrm{Ga})$ As clusters with diameter about $3 \mathrm{~nm}$ with pronounced structural disorder [30]. It is well known that large disorder can lead to drastic reduction of the EXAFS amplitude, and hence, to a lowering of the apparent coordination numbers. It can also cause an apparent contraction (or even expansion) in the nearest neighbor distances, which can be as large as $0.15 \AA$ [31]. Neglecting the presence of disorder and its influence on the results of EXAFS analysis would lead to serious errors. The static disorder is an intrinsic property characteristic for the system. The thermal disorder can be reduced by lowering the temperature, which was done by performing the measurements at liquid nitrogen temperature. To account for the static disorder in the EXAFS fitting procedure for samples ' $a$ ' and ' $c$ ', the coefficients in the cumulant expansion were taken into account in the case in point [32, 33].

To define the local structure changes around Mn atoms, a series of fits were performed. They are marked as fit 1 , fit 2 , and fit 3 in table 2 . The first, fit 1 , was similar to that described in [20]. During the fitting procedure the structural fitting parameters were as follows: the coordination number $N_{\mathrm{As}}$, the coordination shell distance $R_{j}$, the DW factor $\sigma_{j}^{2}$, and $\Delta E_{0 j}$ (edge shift) for each $j$ th coordination shell. The coordination numbers of the second and third coordination shells were constrained to be three times the first shell coordination number, as for the zinc-blende lattice. The results obtained in this work are similar to those presented in [19] 
Table 2. Curve-fitting results for investigated samples.

\begin{tabular}{|c|c|c|c|c|c|c|c|c|}
\hline Shell & Atom & $N$ & $R(\AA)$ & $\sigma^{2}\left(\AA^{2}\right)$ & $C_{3} \times 10^{-4}\left(\AA^{3}\right)$ & $C_{4} \times 10^{-5}\left(\AA^{4}\right)$ & $\mathrm{R}$-factor, $\chi_{v}^{2}$ & Reference \\
\hline \multicolumn{9}{|c|}{ 'as-grown' } \\
\hline 1 & As & 4 & $2.496 \pm 0.003$ & $0.0040 \pm 0.0003$ & & & $0.023,4$ & This work \\
\hline 1 & As & 4 & $2.495 \pm 0.002$ & $0.0050 \pm 0.0003$ & & & $0.015,211$ & [29] \\
\hline 2 & $\mathrm{Ga}$ & 12 & $4.03 \pm 0.01$ & $0.018 \pm 0.003$ & & & & This work \\
\hline 2 & $\mathrm{Ga}$ & 12 & $4.03 \pm 0.02$ & $0.020 \pm 0.002$ & & & & [29] \\
\hline 3 & As & 12 & $4.74 \pm 0.01$ & $0.022 \pm 0.007$ & & & & This work \\
\hline 3 & As & 12 & $4.75 \pm 0.03$ & $0.017 \pm 0.004$ & & & & {$[29]$} \\
\hline \multicolumn{9}{|c|}{$' a$ ' $-500^{\circ} \mathrm{C}$ fit 1} \\
\hline 1 & As & $2.69 \pm 0.45$ & $2.52 \pm 0.01$ & $0.003 \pm 0.001$ & & & $0.047,4.9$ & This work \\
\hline 2 & $\mathrm{Ga}$ & 8.07 & $4.06 \pm 0.05$ & $0.013 \pm 0.003$ & & & & \\
\hline 3 & As & 8.07 & $4.48 \pm 0.07$ & $0.011 \pm 0.004$ & & & & \\
\hline \multicolumn{9}{|c|}{ ' $a$ ' $-500^{\circ} \mathrm{C}$ fit 2} \\
\hline 1 & As & 4 & $2.52 \pm 0.01$ & $0.0057 \pm 0.0005$ & & & $0.074,7.2$ & This work \\
\hline 2 & $\mathrm{Ga}$ & 12 & $4.06 \pm 0.05$ & $0.015 \pm 0.004$ & & & & \\
\hline 3 & As & 12 & $4.47 \pm 0.08$ & $0.013 \pm 0.004$ & & & & \\
\hline \multicolumn{9}{|c|}{$' a$ ' $-500^{\circ} \mathrm{C}$ fit 3} \\
\hline 1 & As & 4 & $2.554 \pm 0.006$ & $0.0078 \pm 0.0003$ & $3.19 \pm 0.52$ & $6.5 \pm 1.7$ & $0.027,2.6$ & This work \\
\hline 2 & $\mathrm{Ga}$ & 12 & $4.07 \pm 0.03$ & $0.014 \pm 0.003$ & & & & \\
\hline 3 & As & 12 & $4.47 \pm 0.05$ & $0.012 \pm 0.003$ & & & & \\
\hline \multicolumn{9}{|c|}{ 'c ${ }^{\prime}-600^{\circ} \mathrm{C}$} \\
\hline 1 & As & 6 & $2.576 \pm 0.002$ & $0.0071 \pm 0.0003$ & & & $0.008,3.2$ & This work \\
\hline 2 & $\mathrm{Mn}$ & 2 & $2.85 \pm 0.01$ & $0.020 \pm 0.001$ & $-3.05 \pm 2.15$ & $34.1 \pm 13.2$ & & \\
\hline 3 & $\mathrm{Mn}$ & 6 & $3.71 \pm 0.01$ & $0.022 \pm 0.001$ & $1.91 \pm 1.90$ & $35.8 \pm 7.60$ & & \\
\hline 4 & As & 6 & $4.52 \pm 0.02$ & $0.009 \pm 0.002$ & & & & \\
\hline 5 & $\mathrm{Mn}$ & 12 & $4.68 \pm 0.02$ & $0.018 \pm 0.002$ & $-5.40 \pm 3.07$ & $25.5 \pm 17.6$ & & \\
\hline 6 & As & 6 & $4.80 \pm 0.06$ & $0.017 \pm 0.010$ & & & & \\
\hline
\end{tabular}

and [20]. A drastic decrease of the coordination numbers was observed. The coordination numbers were changed from 4, 12, and 12 (for the 'as-grown' sample for three coordination shells) to $2.69 \pm 0.45,8.07$, and 8.07 , respectively, for sample ' $a$ '. The reduction of As in the first coordination sphere from 4 to $\sim 2$ is unphysical. It assumes that half of the As atoms are removed from the crystal lattice. Moreover, the DW factors for sample ' $a$ ' (particularly for the first coordination shell) are reduced (which is dubious because of a pronounced structural disorder in the examined sample observed by TEM) [30]. Significant constrictions of the distances for the first (to $2.52 \pm 0.01 \AA$ ) and third $(4.48 \pm 0.07 \AA$ ) shells were found in comparison to the starting model values $2.550 \AA$ and $4.688 \AA$. However, the distance for the second coordination shell is bigger in comparison with the values for starting the ' $\mathrm{Mn}_{\mathrm{Ga}}$ shift' model, $3.998 \AA$, and the 'as-grown' sample, $4.03 \pm 0.01 \AA$. We will return to this issue later.

For the second fit, fit 2 (see table 2), the coordination numbers for three shells $(4,12$, and 12) were taken from the ' $\mathrm{Mn}_{\mathrm{Ga}}$ shift' model and were fixed during the fitting procedure while the rest of the parameters were kept free. It can be clearly seen in figure 8(c) that the quality of the fit is not perfect. The distances of coordination shells are changed in a similar way as for the previous fitting but the values of DW factors are bigger, as was expected. 

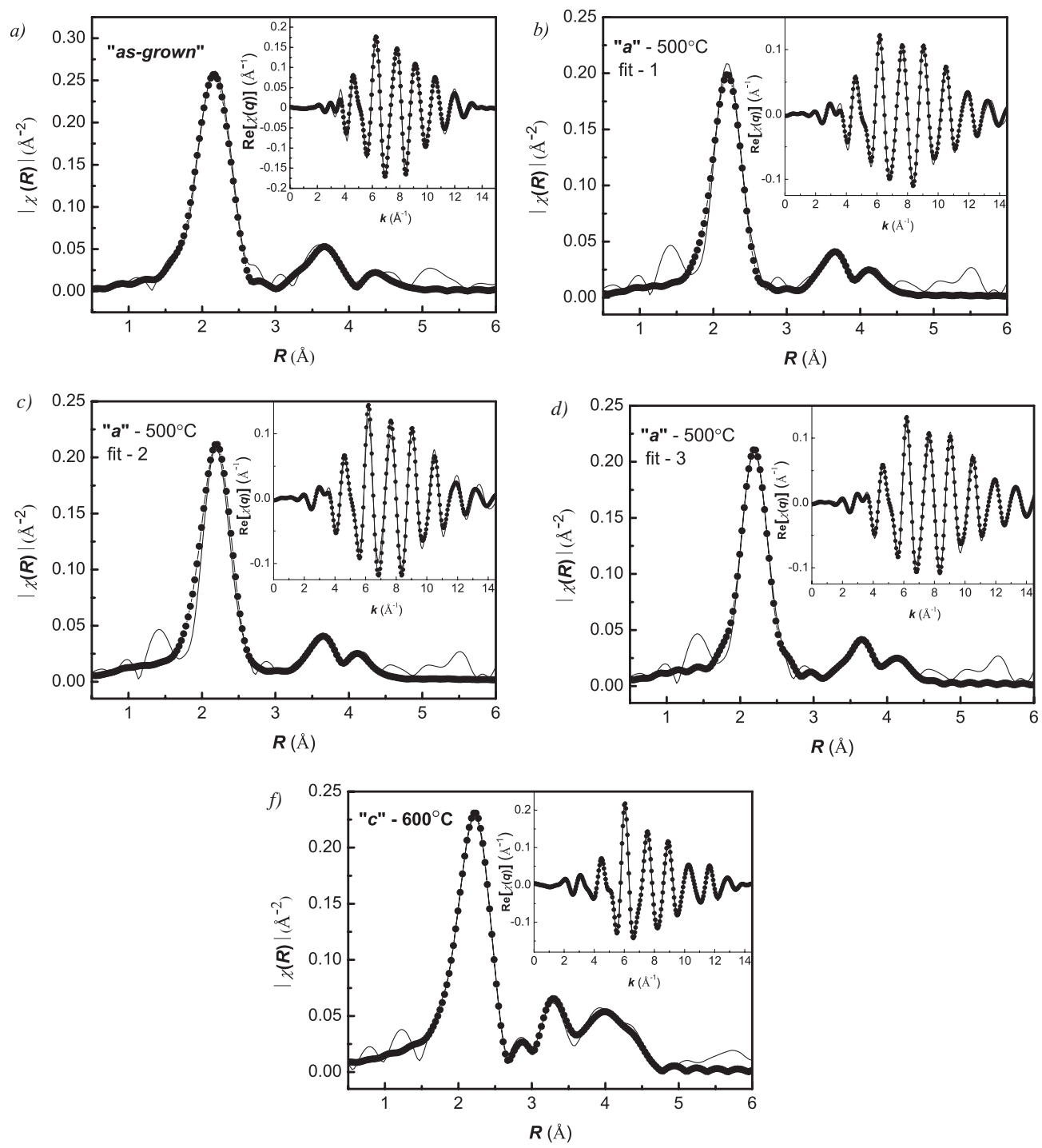

Figure 8. Fourier-transformed EXAFS experimental $k \chi(k)$ spectra (fine lines) compared with the superimposed best fit (circles line) for samples: 'as-grown' $(a)$, ' $a$ ' fit-1 (b), ' $a$ ' fit-2 (c), ' $a$ ' fit-3 $(d)$ (see the text) and ' $c$ ' $(f)$. The least-squares fitting of EXAFS oscillations for three (samples 'as-grown' and ' $a$ ') and six coordination shells (sample ' $c$ ') in k-space is presented in the inset for each fitting. The black line corresponds to the experimental data of the back transform and the circles line represents the fit.

In the third fitting procedure (fit 3, table 2) for the first coordination shell, the coefficients in the cumulant expansion were added. The third $\left(C_{3}\right)$ and fourth $\left(C_{4}\right)$ cumulant describe the deviation of the pair distribution from a Gaussian shape [31]. The third cumulant reflects the degree of asymmetry of the distribution; its positive value accounts for the presence of a tail on the high- $R$ side of the distribution. The positive value of the fourth cumulant indicates a distribution narrower at the center and broader at the borders than a Gaussian one. In figure $8(\mathrm{~d})$ the results of the analysis are presented. One can clearly see that quality of the 
fit is the best from all considered. In table 2, the values of R-factor and reduced chi-square $\left(\chi_{v}^{2}\right)[34]$ (pointing the fit quality) are presented.

Conditions of growth (namely, the growth temperature, the As flux, and the Mn content) strongly influence the $\mathrm{Ga}_{1-x} \mathrm{Mn}_{x}$ As lattice constant. Different values of lattice constants for hypothetical zinc-blende MnAs were reported in the literature, namely: $5.7 \AA$, $5.9 \AA$, and $5.98 \AA[1,2,21,35-40]$. Corresponding Mn-As bond lengths are $\sim 2.47 \AA, \sim 2.55 \AA$, and $\sim 2.59 \AA$. The resulting EXAFS Mn-As bond length for sample ' $a$ ' for the first coordination shell $(2.554 \pm 0.006 \AA)$ is very close to the values corresponding to zinc-blende type MnAs presented in, e.g. [21, 38, 39].

An attempt was made to consider the $\mathrm{Mn}-\mathrm{Mn}$ correlations in the first and second coordination shells to account for the presence of Mn dimers or small clusters of Mn, but it led to unphysical results. This excludes the presence of a significant number of $\mathrm{Mn}$ atoms combined in clusters or precipitates.

For the sample ' $c$ ' annealed at the highest temperature $\left(600^{\circ} \mathrm{C}\right)$, spherical nanocrystals with much bigger diameters (about $20 \mathrm{~nm}$ ) and well-developed MnAs hexagonal structures were observed by TEM [30]. Most of the MnAs clusters were coherent with the GaAs matrix, but some of them revealed weak structural disorder at their boundaries.

To improve the quantitative analysis of sample ' $c$ ' the addition of cumulants was also necessary. Values of the $C_{3}$ and $C_{4}$, which are effective distribution cumulants (for $\mathrm{Mn}-\mathrm{Mn}$ coordinations), clearly established the presence of large disorder in the Mn-Mn distances. The results of this analysis are presented in table 2 and in figure 8(f). Qualitative and quantitative analysis of EXAFS spectra for sample ' $c$ ' confirmed the new phase formation, namely, MnAs hexagonal precipitates. The distances for the six nearest coordination shells are in good agreement with 'MnAs' model values (see table 1) and those reported in the literature for MnAs compound [41].

Knowing the atomic structure of a sample, let us now relate it to its magnetic properties. The magnetic properties of $\mathrm{Ga}_{1-x} \mathrm{Mn}_{x}$ As as-grown samples, within wide limits of $x$, have been actively studied, e.g. [42]. The Curie temperature for the $\mathrm{Ga}_{1-x} \mathrm{Mn}_{x}$ As layer with $x=0.053$ was reported to be about $110 \mathrm{~K}$ [9]. The magnetic properties of samples ' $a$ ' and ' $c$ ' are presented in figure 9 with the ferromagnetic contribution clearly evidenced by magnetization hysteresis loops. For sample ' $c$ ', annealed at the highest temperature, the ferromagnetic state exists up to room temperature. The saturation value of magnetization $M_{\mathrm{s}}$ found experimentally in this layer is about $20 \mathrm{emu} \mathrm{cm}^{-3}$ at $T=10 \mathrm{~K}$. It agrees well with the results of previous magnetic studies of $\mathrm{Ga}_{1-x} \mathrm{Mn}_{x}$ As layers annealed at high temperatures: $M_{\mathrm{s}}=17 \mathrm{emu} \mathrm{cm}{ }^{-3}$ was observed in [43] in the layer with $x=0.05$, while $M_{\mathrm{s}}=40 \mathrm{emu} \mathrm{cm}^{-3}$ was found in [44] in the layer with $x=0.06$. Assuming the expected magnetic moment of 5 Bohr magnetons per Mn ion and the technological data for Mn content and layer thickness, one can estimate the magnetization saturation value $M_{\mathrm{s}}=50 \mathrm{emu} \mathrm{cm}{ }^{-3}$ for an ideal ferromagnetic $\mathrm{Ga}_{1-x} \mathrm{Mn}_{x}$ As layer with $x=0.05$. Taking into account the unavoidable reduction of the magnetization expected due to non-collinear spins at the surface of nanoclusters and magnetic blocking effects, one can conclude that a large portion of Mn ions present in the layer belongs to the ferromagnetic nanocluster phase. It strongly supports the conclusion of the structural analysis of annealed layer ' $c$ ', evidencing the presence of hexagonal MnAs inclusions for which a Curie temperature of about $310 \mathrm{~K}$ is expected [43, 44]. The magnetic properties of the layer annealed at lower temperature (figure 9(a)) show about 10 times smaller ferromagnetic contribution characterized by much smaller coercive field. Based on the results of the XRD and EXAFS analysis, we attribute this behavior to the formation stage of Mn-rich zinc-blende clusters within the GaMnAs layer exhibiting strong magnetic disorder and a broad ferromagnetic transition region. The largest portion of Mn ions in annealed GaMnAs layer ' $a$ ' is in the paramagnetic state. 

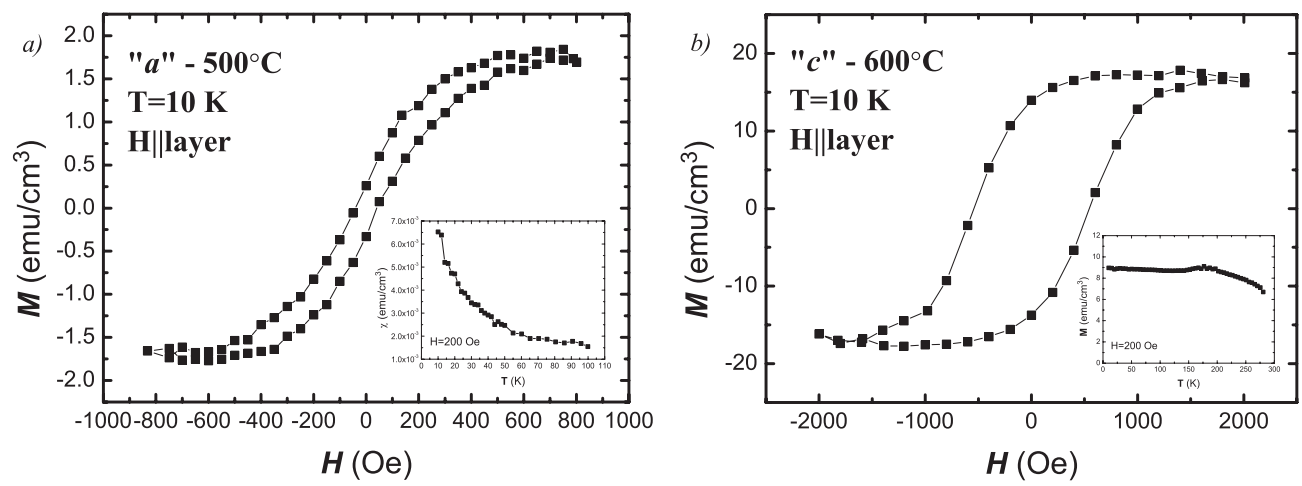

Figure 9. Magnetic hysteresis loops of annealed $\mathrm{Ga}_{0.95} \mathrm{Mn}_{0.05} \mathrm{As}$ layers ' $a$ ' and ' $c$ '. The insets show the temperature dependence of the magnetization.

In summary, the EXAFS investigation proved that after thermal treatment, the local structure transition around $\mathrm{Mn}$ atoms takes place from the chemically homogeneous ' $a$ s-grown' layer to the chemically inhomogeneous layer (with cubic $\mathrm{Ga}(\mathrm{Mn}) \mathrm{As}$ clusters, sample ' $a$ '), and further to the material (sample ' $c$ ') with well-developed MnAs hexagonal clusters. This points out that the investigated structure is unambiguously placed in the groups described in [45]. It is worth emphasizing that high temperature annealing $\left(600^{\circ} \mathrm{C}\right)$ results in a dramatic modification of the magnetic properties of the layer with a Curie temperature of about $300 \mathrm{~K}$, which is the crucial point for spintronic materials applications.

\section{Conclusions}

The influence of high temperature annealing on the structural and magnetic properties of GaMnAs layers grown by MBE was investigated by EXAFS, HRXRD, and SQUID techniques. It was shown that annealing at $500{ }^{\circ} \mathrm{C}$ introduces large disorder in the zinc-blende GaMnAs structure with $\mathrm{Mn}$ concentration of 0.05 and causes the relaxation of the matrix from highly compressively strained in the 'as-grown' sample to only slightly strained. Simultaneously the structure around $\mathrm{Mn}$ atoms becomes more disordered as compared to the reference sample and the distance between $\mathrm{Mn}-\mathrm{As}$ atoms in the first coordination sphere becomes much longer and is close to that predicted for MnAs cubic structure $(\sim 2.55 \AA)$. No evidence for the formation of Mn dimers or small clusters was found. The annealing at $600^{\circ} \mathrm{C}$ leaves small tensile strain in the GaAs host matrix but results in the formation of hexagonal MnAs inclusions, which still have large structural disorder and clearly formed hexagonal structure. The MnAs inclusions are ferromagnetic with a Curie temperature of about $300 \mathrm{~K}$. TEM studies performed for the same sample, studied by EXAFS and described in this paper, revealed that the hexagonal inclusions of MnAs had a spherical shape with diameter around $20 \mathrm{~nm}$. Most of the MnAs clusters were coherent with the GaAs matrix. The annealing of $(\mathrm{Ga}, \mathrm{Mn}) \mathrm{As}$ at $600^{\circ} \mathrm{C}$ is a suitable method to form small hexagonal MnAs inclusions in the GaAs host matrix, exhibiting ferromagnetic properties at room temperature.

\section{Acknowledgments}

We would like to thank W Paszkowicz for helpful discussions. This work was partially supported by a national grant from the Ministry of Science and High Education N202-05232/1189 and by DESY and the European Community under Contract RII3-CT-2004-506008 (IA-SFS). 


\section{References}

[1] Ohno H, Shen A, Matsukura F, Oiwa A, Endo A, Katsumoto S and Iye Y 1996 Appl. Phys. Lett. 69363

[2] Ohno H 1998 Science 281951

[3] Hayashi T, Tanaka M, Nishinaga T and Shimada H 1997 J. Appl. Phys. 814865

[4] Dietl T, Ohno H, Matsukura F, Cibert J and Ferrand D 2000 Science 2871019

[5] Macdonald A H, Schiffer P and Samarth N 2005 Nat. Mater. 4195

[6] Gleason J N, Hjelmstad M E, Dasika V D and Goldman R S 2005 Appl. Phys. Lett. 86011911

[7] Sanvito S and Hill N A 2001 Appl. Phys. Lett. 783493

[8] Yu K M, Walukiewicz W, Wojtowicz T, Lim W L, Liu X, Bindley U, Dobrowolska M and Furdyna J K 2003 Phys. Rev. B $68041308(\mathrm{R})$

[9] Ohno H and Matsukura F 2001 Solid State Commun. 117179

[10] Xu J L, van Schilfgaarde M and Samolyuk G D 2005 Phys. Rev. Lett. 94097201

[11] Nazmul A M, Amemiya T, Shuto Y, Sugahara S and Tanaka M 2005 Phys. Rev. Lett. 95017201

[12] Chiba D, Nishitani Y, Matsukura F and Ohno H 2007 Appl. Phys. Lett. 90122503

[13] Campion R P, Edmonds K W, Zhao L X, Wang K Y, Foxon C T, Gallagher B L and Staddon C R 2003 J. Cryst. Growth 251311

[14] Ohya S, Ohno K and Tanaka M 2007 Appl. Phys. Lett. 90112503

[15] Ohno H 1999 J. Magn. Magn. Mater. 200110

[16] Shimuzu H, Miyamura M and Tanaka M 2000 J. Vac. Sci. Technol. B 182063

[17] Soo Y L, Kioseoglou G, Kim S, Chen X, Luo H, Kao Y H, Sasaki Y, Liu X and Furdyna J K 2002 Appl. Phys. Lett. 802654

[18] Soo Y L, Kioseoglou G, Kim S, Chen X, Luo H, Kao Y H, Lin H-J, Hsieh H H, Hou T Y, Chen C T, Sasaki Y, Liu X and Furdyna J K 2003 Phys. Rev. B 67214401

[19] Soo Y L, Wang S, Kim S, Kim G, Cheon M, Chen X, Luo H, Kao Y H, Sasaki Y, Liu X and Furdyna J K 2003 Appl. Phys. Lett. 832354

[20] d'Acapito F, Smolentsev G, Boscherini F, Piccin M, Bais G, Rubini S, Martelli F and Franciosi A 2006 Phys. Rev. B 73035314

[21] Shioda R, Ando K, Hayashi T and Tanaka M 1998 Phys. Rev. B 581100

[22] Adell M, Ilver L, Kanski J, Stanciu V, Svedlindh P, Sadowski J, Domagala J Z, Terki F, Hernandez C and Charar S 2005 Appl. Phys. Lett. 86112501

[23] Moreno M, Jenichen B, Kaganer V, Braun W, Trampert A, Däweritz L and Ploog K H 2003 Phys. Rev. B 67235206

[24] Ravel B and Newville M 2005 J. Synchrotron Radiat. 12537

[25] Zabinsky S I, Rehr J J, Ankudinov A, Albers R C and Eller M J 1995 Phys. Rev. B 522995

[26] Ankudinov A L, Ravel B, Rehr J J and Conradson S D 1998 Phys. Rev. B 587565

[27] Lee P A, Citrin P H, Eisenberger P and Kinkaid B M 1981 Rev. Mod. Phys. 53769

[28] Ravel B 2001 EXAFS Analysis with FEFF and FEFFIT, Path 2: Commentary http://cars9.uchicago.edu/ ravel/ talks/course/notes.pdf

[29] Stuckey A M 2005 X-ray and studies of Mn doped III-V materials Dissertation University of Notre Dame

[30] Kwiatkowski A, Borysiuk J, Bożek R, Wasik D, Kamińska M, Sadowski J and Twardowski A 2006 Acta Phys. Polym. A 110233

[31] Teo B K 1986 EXAFS: Basic Principles and Data Analysis (Berlin: Springer)

[32] Rehr J J and Albert R C 2000 Rev. Mod. Phys. 72621

[33] Bunker G 1983 Nucl. Instrum. Methods Phys. Res. 207437

[34] Newville M 1998 FEFFIT: Using FEFF to model XAFS data http://cars9.uchicago.edu/ifeffit/Documentation

[35] Hong H-M, Kang Y-J, Kang J, Lee E-C, Kim Y-H and Chang K J 2005 Phys. Rev. B 72144408

[36] Hayashi T, Tanaka M, Nishinaga T and Shimada H 1997 J. Cryst. Growth 175/176 1063

[37] Furdyna J K and Kossut J 1986 Superlatt. Microstruct. 289

[38] Kuryliszyn-Kudelska I, Domagala J Z, Wojtowicz T, Liu X, Lusakowska E, Dobrowolski W and Furdyna J K 2004 J. Appl. Phys. 95603

[39] Shirai M, Ogawa T, Kitagawa I and Suzuki N 1998 J. Magn. Magn. Mater. 177-181 1383

[40] Schott G M, Faschinger W and Molenkamp L W 2001 Appl. Phys. Lett. 791807

[41] Soo Y L, Huang S W, Ming Z H, Kao Y H, Munekata H and Chang L L 1996 Phys. Rev. B 534905

[42] Oiwa A, Katsumoto S, Endo A, Hirasawa M, Iye Y, Ohno H, Matsukura F, Shen A and Sugawara Y 1997 Solid State Commun. 103209

[43] Kwiatkowski A, Wasik D, Kamińska M, Bozek R, Szczytko J, Twardowski J A, Borysiuk J, Sadowski J and Gosk J 2007 J. Appl. Phys. 101113912

[44] Moreno M, Trampert A, Jenichen B, Daweritz L and Ploog K H 2002 J. Appl. Phys. 924672

[45] Ohno H 2006 Phys. Status Solidi c 34057 